\title{
The Protective Role of Parent Resilience on Mental Health and the Parent-Child Relationship During COVID-19
}

\author{
Beth S. Russell ${ }^{1}\left[\right.$ - Alexandria J. Tomkunas ${ }^{1} \cdot$ Morica Hutchison $^{1} \cdot$ Rachel R. Tambling $^{1} \cdot$ Abagail L. Horton $^{1}$
}

Accepted: 5 September 2021 / Published online: 17 September 2021

(c) The Author(s), under exclusive licence to Springer Science+Business Media, LLC, part of Springer Nature 2021

\begin{abstract}
The COVID-19 pandemic is linked to particularly potent psychological effects for children and their caregivers while families adjust to new daily routines for work, education, and self-care. Longitudinal associations are presented from a national sample of 271 parents (mean age $=35.29$ years, $48.5 \%$ female) on resilience, mental health and stress indicators, and parenting outcomes. Multigroup path model results indicate significant associations between resilience and parent stress or parent perceived child stress initiates a sequence of significant linkages to parent depression, followed by caregiver burden and parent-child relationship quality. This final set of linkages between depression and both parenting outcomes were significantly stronger for men, who also reported higher rates of perceived child stress. Results suggest that fathers' depression symptoms and associated spill-over to perceived child stress is producing stronger effects on their parenting experiences than effects reported by mothers.
\end{abstract}

Keywords Resilience $\cdot$ Caregiver burden $\cdot$ Parenting $\cdot$ COVID-19

\section{Introduction}

In the United States, the COVID-19 (SARS-COV-2) global pandemic led to shelter-in-place orders, school and workplace closures, and widespread quarantine beginning in March of 2020, dramatically impacting individuals, families, and communities. The mental health sequalae of disasters like the present pandemic are extensive and widespread [1, 2], and symptoms may result from the steps taken to slow the spread of the virus. Efforts to mitigate the spread of the disease created pervasive and prolonged disruptions to family routines and daily life [3]. For example, social distancing practices and quarantines may be crucial for containing disease prevalence, but are also associated with lasting mental health impacts [4]. Early research during the COVID-19 pandemic indicates heightened stressors of uncertainty of the future, fear of infection, economic vulnerability, and limited access to reliable resources and information, all of which have negative psychological impacts on individual

Beth S. Russell

Beth.Russell@uconn.edu

1 Department of Human Development \& Family Sciences, University of Connecticut, 348 Mansfield Rd. U-1058, Storrs, CT 06269, USA and family wellbeing [5, 6]. As a result, the COVID-19 pandemic is an instance of traumatic stress, creating pathogenic conditions for families that may endure for months to come [7]. In this study, we examine longitudinal impacts on parent-child relationships and caregiver burden in the context of parents' reports of resilience, stress (both general and COVID-specific), depression and anxiety, and closeness and conflict with their child.

Families' abilities to adapt to the stresses that unfold over the weeks and months of an enduring community-wide crisis like a pandemic rely on the ability to activate individual and contextual resources to maintain wellbeing for all family members. Adult resilience is somewhat understudied in comparison to the volume of research on children and youth [8], nevertheless, studies indicate adults' abilities to adapt positively to stressors following traumatic events are more common than not,this is particularly true among those who do not have pre-existing mental health symptoms that impact developing healthy and sustainable coping behaviors [9]. Of those adults who experience negative effects following a disaster, several characteristics are noted to increase the risk of negative outcomes: being female, belonging to an ethnic minority group, having existing mental health symptoms, and the presence of additional stressors or unstable resources [10]. Of particular interest to this paper is the influence of 
gender on adult resilience. Similar to results reported by Norris et al. [10] following 9/11, Bonanno et al. [11] found women were less likely to be resilient than men.

In order to respond to such stressors adaptively, an individual relies on protective environmental resources and individual skills $[12,13]$. Thus, resilience is a process and is not solely a product of intrinsic characteristics, resilient adaption to stressors requires the interaction of personal abilities, relationships, and contextual factors [14]. The work of resilience researchers highlights the multi-level nature of resilience, emphasizing the influence of ecological systems on individual characteristics over time $[8,15,16]$. An individual's capacity to be resilient in the face of adversity is dependent on protective factors as they relate to particular outcomes for a given timeframe, at both the individual and environmental levels. For example, the stressors experienced during COVID-19 may vary from those felt just a few months earlier, and they may continue to evolve as the prevalence rates of COVID-19 diagnoses surge and recede. Additionally, the resources mustered to manage these stressors may also be dynamic and specific to the COVID-19 context as the pandemic unfolds. Longitudinal examinations of resilience and its impacts over time are needed to better understand for whom and under what circumstances resilience may buffer individuals and their families from the negative impacts of stressful events; this is particularly true during a community-wide and enduring stressful crisis like the COVID-19 pandemic.

\section{Parent Mental Health and Caregiving During a Crisis}

The negative mental health impacts from a wide range of disasters-including natural hazards and manmade eventscan lead to prolonged periods of increased psychiatric symptomology, including anxiety and depression [17-21]. During epidemic conditions, quarantine-related stressors that impact these mental health outcomes include prolonged duration of isolation, infection fears, frustration, boredom, inadequate supplies and information, financial loss, and stigma [5, 22]. Further, periods of uncertainty with an indeterminant endpoint $[23,24]$, including quarantines, constitute a stressful experience with particular salience for families with children [25]. Evidence suggests that compared to their non-caregiving counterparts, parents experience more acute negative disaster responses $[18,26,27]$. This may be because parents shoulder additional caregiving burdens during disasters, which may heighten anxiety and posttraumatic stress [28, 29]. Specifically, parents not only carry responsibility for cultivating their own coping and self-care responses during a crisis, but as a primary source of children's coping socialization, they also shape disaster outcomes for their children by modeling coping behavior [30, 31]. Studies of the linkages between parents' behavior and children's outcomes following disasters reports worse child outcomes among children of highly distressed parents [32, 28, 33, 34]. The consequences of millions of families sheltering in place for an undefined period of time may lead to unprecedented impacts from the COVID-19 pandemic on individual health and family well-being. Many of the structures relied on for support in challenging times are less accessible than in any other recent times-be those supports family gatherings, schools, community health or religious centers. As of this writing, eight months into the pandemic in the United States, rates of COVID-19 diagnoses are surging across the country, setting new records beyond the initial peak of the disease recorded on April 10, 2020 [35], communities are struggling to develop flexible work and school re-opening plans that are sensitive to infection risk and provide the best possible childcare and education supports for families with children. Concurrently, the economic impacts from the pandemic are escalating such that rapidly changing unemployment rates have recently hit record highs above 14\% [36].

As noted by previous researchers [26, 27] meeting the unprecedented demands of parenting during COVID-19 forces parents to develop new caregiving, work, and education routines. The complexity of parents' roles is well described by Family Systems' theory and parenting research, which illustrate parents' need to attend and respond to the care of multiple family subsystems (e.g., individual selfcare, coordination of co-parenting demands, and parenting needs); [37-39]. High levels of psychological distress and caregiver burden during a disaster may complicate the tension between these parallel needs for care [40, 41]. Further, parents' mental health symptoms may interfere with parents' perceptions of their children's stress, as seen in depressed and anxious mothers' overreporting of their children's psychiatric symptoms [42], and in withdrawn or unavailable patterns of parent-child interaction [43-45]. Adding an additional risk consideration is the presence of pre-existing mental health symptoms that may hamper parents' abilities to leverage the necessary resources to be resilient to the challenges presented by COVID-19, including coping with the strains of caregiver burden [46].

Taken together, the literature summarized thus far suggests that the stresses and strains of parenting during disasters may amplify mental health symptoms, potentially taxing parents' resilience sufficiently to impact the perceived closeness or patterns of conflict within the parent child relationship — these relationship qualities constitute a significant predictor of outcomes for children exposed to prolonged periods of stress [28, 33, 34]. For example, children's reports of conflict with their parents following a disaster is positively associated with children's post-traumatic stress symptoms [47]. While these findings highlight the potential 
negative consequences for parents and their relationships with their children during the COVID-19 pandemic, not all children whose parents experience mental health symptoms will face maladaptive outcomes [48]. Additionally, transient intervals of less responsive parenting may not adversely impact parent-child relationships or the emotional tenor of the home $[49,50]$. Unanswered questions arise, then, concerning the extent to which parents' resilience is taxed by mental health symptoms and by relational factors that ultimately influence parents' experience as caregivers, including the quality of their relationships with their children during this unprecedented, stressful time.

\section{Current Study}

The present effort concerned the associations between resilience, mental health and stress indicators, and parenting outcomes (caregiver burden and parent-child relationship qualities) over a 30-day period during the COVID-19 pandemic. Using data from a national sample of parents approximately five weeks after the first U.S. quarantines were advised, we explore the longitudinal associations between these factors through multigroup path analysis. Our research questions include whether there are significant associations from resilience to the mental health and stress indicators influenced by the COVID-19 pandemic on parenting outcomes? Given evidence from Norris et al., [10] that individual characteristics including gender are associated with resilience, how would mothers' and fathers' experiences of caregiver burden and parent-child relationship quality vary? Informed by evidence of the protective influence of parents' resilience [9, 10], we hypothesize that baseline resilience would be negatively associated with parents' stress, anxiety and depression symptoms. Further, based on previous evidence that these mental health experiences can shape parents' perceptions of their children [26, 51], we anticipate subsequent associations between parents' perceptions of their children's stress. Lastly, we expect each of these stress and mental health variables would also be associated with parenting experience, such that we would anticipate positive associations with parent-child conflict and caregiver burden and negative associations with parent-child closeness.

\section{Methods}

Data presented here include longitudinal survey results collected from April 27-28, 2020 (baseline), and followup data collected 30 days later. All authors certify responsibility for the study including analysis and interpretation of data leading to the presentation of these results, and have no known conflicts of interest to report.

\section{Participants}

Individuals who were 18 years or older, living in the U.S., who speak English and were caring for a child under 18 years old in their home were eligible to participate through Amazon's online worker pool, Mechanical Turk (MTurk). MTurk workers are reported as more diverse, but otherwise fairly representative of the characteristics of larger online populations, including the U.S. [52, 53], a small body of recent evidence suggests this population scores slightly higher on depression inventories, but comparably to the broader general population on indices of personality [54-56]. 271 parents of minors provided complete data for all key variables of interest (see measures below) at baseline and 189 at the 30-day follow up. Of these, $124(48.5 \%)$ of participants were female, and 97 $(35.8 \%)$ reported belonging to a racial minority group and $63(23.2 \%)$ identified as LatinX. Caregivers reported an average age of 35.29 years ( range $=19-72$ years old) and were asked to report the age of the focal child in the following categories: birth to 5 years old $(n=103,38 \%)$, 6 to 11 years old $(\mathrm{n}=95,35.1 \%)$, and 12 to 18 years old $(\mathrm{n}=71,26.4 \%$,See Table 1 for further demographic details).

\section{Procedures}

All study materials were approved by the University of Connecticut IRB (X20-0075) prior to recruitment from MTurk's online worker pool for participation in the anonymous study of family experiences and coping during the COVID-19 pandemic. Participants who consented to participate in the study and met inclusion criteria were able to complete the survey set and were compensated for their time. MTurk has shown recruitment and data collection to be both replicable and valid [53, 57], but given concerns about the validity of data drawn through crowd-sourced convenience samples [58], rigorous data management practices were followed. Specifically, to verify the inclusion of unique individual human respondent cases (as opposed to computerized bot responses) and the attentiveness of each response, we screened the dataset for duplicate cases by worker ID and global positioning verification within the United States. We also screened cases on the basis of a response time analysis, such that we eliminated any response received in less than $3.4 \mathrm{~min}$ or $10 \%$ of the average completion time of $34 \mathrm{~min}$ (based on pilot efforts with the survey battery).

\section{Measures}

Participants were asked to answer questions about demographic characteristics such as gender, sexual orientation, 
Table 1 Demographic characteristics at baseline

\begin{tabular}{|c|c|c|c|}
\hline & $\begin{array}{l}\text { Overall sample } \\
\mu(\mathrm{SD})\end{array}$ & $\begin{array}{l}\text { Female caregivers } \\
\mu(\mathrm{SD})\end{array}$ & $\begin{array}{l}\text { Male caregivers } \\
\mu \text { (SD) }\end{array}$ \\
\hline \multirow[t]{2}{*}{ Age } & $35.29(8.35)$ & $36.97(8.41)$ & $33.88(8.06)$ \\
\hline & $\mathrm{N}(\%)$ & $\mathrm{N}(\%)$ & $\mathrm{N}(\%)$ \\
\hline \multicolumn{4}{|l|}{ Gender } \\
\hline Male & $147(54.2 \%)$ & - & $147(100 \%)$ \\
\hline Female & $124(45.8 \%)$ & $124(100 \%)$ & - \\
\hline \multicolumn{4}{|l|}{ Race } \\
\hline Black/African American & $25(9.2 \%)$ & $10(8.1 \%)$ & $15(10.2 \%)$ \\
\hline Asian/Asian American & $39(14.4 \%)$ & $9(7.3 \%)$ & $30(20.4 \%)$ \\
\hline Native Hawaiian/Other Pacific Islander & - & - & - \\
\hline American Indian/Alaska Native & $21(7.7 \%)$ & $9(7.3 \%)$ & $12(8.2 \%)$ \\
\hline White & $174(64.2 \%)$ & $92(74.2 \%)$ & $82(55.8 \%)$ \\
\hline \multicolumn{4}{|l|}{ Ethnicity } \\
\hline LatinX & $63(23.2 \%)$ & $16(12.9 \%)$ & $47(32 \%)$ \\
\hline Non-LatinX & $208(76.8 \%)$ & $108(87.1 \%)$ & $100(68 \%)$ \\
\hline \multicolumn{4}{|l|}{ Partner status } \\
\hline Partnered/married & $233(86 \%)$ & $97(78.2 \%)$ & $136(92.5 \%)$ \\
\hline Non-partnered & $38(14 \%)$ & $27(21.8 \%)$ & $11(7.5 \%)$ \\
\hline \multicolumn{4}{|l|}{ Focal child age category } \\
\hline Birth to 5 years old & $103(38 \%)$ & $36(29 \%)$ & $67(45.9 \%)$ \\
\hline 6 to 11 years old & $95(35.1 \%)$ & $47(37.9 \%)$ & $48(32.7 \%)$ \\
\hline 12 to 18 years old & $71(26.4 \%)$ & $40(32.3 \%)$ & $31(21.1 \%)$ \\
\hline \multicolumn{4}{|l|}{ Finances adequate to meet needs } \\
\hline Not met & $125(46.1 \%)$ & $62(50 \%)$ & $63(42.9 \%)$ \\
\hline Met & $146(53.9 \%)$ & $62(50 \%)$ & $84(57.1 \%)$ \\
\hline
\end{tabular}

race/ethnicity, caregiver age, focal child age on whom participants based their answers for key variables, financial security ("do you have enough money to meet your needs", rated from $1=$ not at all to $5=$ completely), and partner status (non-partnered: single, divorced or widowed, or partnered: married or living with a significant other). Additional details on the sample are presented in Table 1.

\section{Resilience}

The Adult Resilience Measure (ARM; [59] is a 17-item measure that assesses the personal and relational resilience of an adult using a 5-point Likert scale ranging from 1 "Not at all" to 5 "A lot." The ARM has two subscales-personal and relational resilience. 10 items are summed for a score of personal resilience, ranging from 10 to 50, and 7 items are summed for a score of relational resilience, ranging from 7 to 35 ; higher scores indicate greater resilience in both cases. The reported Cronbach's alphas are good $(\alpha=0.88$ for both personal and relational subscales, [59], and were equally strong in the present study ( $\alpha=0.87$ and 0.90 for personal and relational subscales, respectively).

\section{Perceived Stress}

The Perceived Stress Scale-Adult form (Parent Perceived Stress; [60] is a 10-item measure that uses a 5-point Likert scale (from "Never" to "Very often") to evaluate participant's thoughts and feelings about their personal stress during the past two weeks. Items are summed to create a total score ranging from 0 to 40 ,higher scores indicate increased perceived stress. Stress level categories for this scale include: 0-13 indicates mild stress, 14-26 indicates moderate stress, and 27-40 indicates high perceived stress. The reported Cronbach's alpha is good $(\alpha=0.85$; [60], as are those for current sample at baseline $(\alpha=0.86)$.

\section{Perceived Child Stress}

The Perceived Stress Scale-Child form was used from the National Institutes of Health Toolkit's emotion resources [61],This 10-item version of the original Perceived Stress Scale [60] adapted by Cohen to asked parents to report their perceptions of their child's general stress using a 5-point Likert scale, from "Never" to "Very often" during the past two weeks. Items are totaled for a score ranging from 0 to 40; higher scores indicate higher perceived stress. The 
reported Cronbach's alpha from a sample of parents of children as young as eight years of age are good $(\alpha=0.87$; [62], as are those for the current sample $(\alpha=0.83)$.

\section{COVID-19-specific Stressors-Family items}

A disaster-specific measure of stressors (COVID-stressors; [63] was adapted for use to assesses parents' exposure to and appraisal of stressors specific to the COVID-19 pandemic. The original measure assesses past-week exposure to 23 stressors across three conceptual groupings: Infectionrelated stressors, Daily Activity stressors, and Financial/ Resource-related stressors. An additional, fourth group of six Family-focused items were developed for this study adapted from the Child Routines Inventory [64] to assess parents' stress due to changes in children's daily routines. The items included three from the original Sytsma et al. [64] measure: children's daily living routines, household responsibilities, and discipline routines, as well an adapted version of a fourth item wherein "homework routines" was modified to read "education routines" to include online learning during the pandemic. Additionally, researchers created two items to capture stress due to 1) changes in children's social routines and 2) interactions parents had with their children. These adaptations resulted in a total of 29 stressor items. Consistent with the original measure, participants are first asked to endorse the stressors they have experienced by responding to a binary question "did you experience stressor $X$ ?". For each affirmatively endorsed stressor, respondents are prompted to rate the stressfulness of the item on a scale of 1 to 5 ("not at all stressful" to "extremely stressful"). A total stressor count of the items endorsed, as well as total stress appraisals for the continuous ratings of stressfulness can be calculated [63], the current study included total appraisal scores in the analysis presented below. The preliminary psychometric evaluation of the 23-item measure indicates a unidimensional structure and high internal consistency (Cronbach's alpha $=0.96$; [65]. The adapted version used in this study performed equally well at baseline, with a Cronbach's alpha of 0.90; additionally, the new familyspecific subset of stressors also had good internal consistency at baseline, with an alpha of 0.86 .

\section{Anxiety}

Generalized Anxiety Disorder-7 (GAD-7; [66] is a 7-item scale that evaluates the extent of generalized anxiety symptoms and the associated disorder by rating the frequency of anxiety symptoms over the past two weeks using a 5-point Likert scale ranging from "Not at all" to "Nearly every day". Items are summed to create a total score ranging from 0 to 21 , higher scores indicate greater presence of anxiety symptoms. Categories of levels of anxiety for this scale include:
0-5 for minimal anxiety, 5-9 for mild anxiety, 10-14 for moderate anxiety, and 15-21 for severe anxiety [66]. The reported Cronbach's alphas are good $(\alpha=0.90$; [66], and slightly stronger in the current sample $(\alpha=0.92)$.

\section{Depression}

The Major Depression Inventory (MDI; [67]) is a 12-item scale that evaluates depressive symptoms using a 6-point Likert scale ranging from "At no time" to "All of the time". Items are summed to create a total score ranging from 0 to 50 that represents general severity of depression symptoms, higher scores represent greater presence of depressed symptoms. Responses can be aligned with diagnostic criteria to establish if participants currently meet criteria for a major depressive episode [68]. Categories of levels of depression for this scale include: 20-24 indicating mild depression, 25-29 indicating moderate depression, and 30-50 indicating severe depression [69]. The reported Cronbach's alpha is excellent at 0.94 [67], just as in the current sample $(\alpha=0.95)$.

\section{Caregiver Burden}

The Burden Scale for Family Caregivers-short form (BSFC$\mathrm{s}$; [70] is a 10-item measure that uses a 4-point Likert scale ranging from "Strongly disagree" to "Strongly agree" to evaluate the perceived burden of caregivers' responsibilities during the past two weeks. Items are summed to create a composite score ranging from 0 to 30 ,higher scores indicate heightened burden levels. Interpretation of burden levels for this scale include: 0-4 indicating mild to no burden, 5-14 indicating moderate burden, and scores from 15 to 30 indicate severe to very severe burden. The reported Cronbach's alphas are excellent $(\alpha=0.92,[70]$, as in the present study $(\alpha=0.94)$.

\section{Relationship Quality}

The Child-Parent Relationship Scale (CPRS; [71] is a 15-item scale that uses a 5-point Likert scale ranging from "Definitely does not apply" to "Definitely applies" to evaluate parents' perceptions of their relationships with their child. The CPRS contains two subscales, an 8-item conflict subscale that evaluates the parents' perceived negativity in the parent-child relationship, and a 7-item closeness subscale that evaluates the parents' perception of the warmth, affection and open communication. The scale is scored by totaling items on the two subscales such that higher scores indicate increased perceived conflict or closeness. The reported Cronbach's alpha from samples of parents of children as young as 3 years of age for the two subscales are acceptable $(\alpha=0.83$ and 0.72 for conflict and closeness, 
respectively; [71] and are good in the current sample ( $\alpha=0.88$ and 0.84 for conflict and closeness, respectively).

\section{Analyses}

\section{Path Analyses}

The multigroup path analysis model was developed in SPSS AMOS [72] using maximum likelihood estimation. The hypothesized path model examined both direct and indirect associations among caregiver's baseline individual resilience, individual perceived stress, perceived child stress, the family-focused COVID- specific stressors and stress appraisals, depression symptomology, and caregiver burden and child-parent relationship conflict at the 30-day followup. Based on evidence that resilience, coping and parenting experiences may vary by gender generally and specifically in the context of COVID-19 [10, 27, 73, 74], a single model, multi-group path analysis was fit to the data organized by parent gender (male/female groups). Given developmental shifts in parenting, focal child age category ( 0 to 5,6 to 11 , and 12 or more years of age) was used as a control.

The model was evaluated by considering data from several fit indices to ensure fit was evaluated fairly and without temptation to only select the fit indices that provided the best model interpretation. Hayduk et al. [75] caution against strict cutoffs of fit indices, as these are often misapplied, while Barrett [76] argues against interpretation of fit indices other than the Chi square, as they are impacted by a number of factors in addition to model specification. Given the discrepancy in the field of which fit indices to select, we elected to examine the following indices with associated cut off values: Model Chi Square $p$ value of less than 0.05; CFI 0.90 for good fit [77], Root Mean Square Error of Approximation $<0.08$ for acceptable fit and $<0.05$ for good fit (RMSEA; [78].

\section{Results}

\section{Descriptive Results and Bivariate Associations}

Means for variables of interest were calculated to determine proportions of caregivers' responses that fell above or below relative reported cutoffs. Parents reported an average individual stress score of 16.12, and a perceived child stress score of 23.96 - which is above the $50^{\text {th }}$ percentile of scores and indicates moderate stress [60]. The sample average for depression score of 19.49 , indicating scores just below the 20-point cut-off for mild depression [69]. Parents reported an average caregiver burden score of 12.69 at the 30-day follow up, indicating moderate levels of burden [70]. The average scores for child parent relationship conflict and closeness in this sample were 16.59 and 30.23 , indicating below average conflict and above average closeness given the potential range of scores [71].

Bivariate associations (see Table 2) indicate caregiver burden at the 30-day follow-up was significantly negatively associated with baseline individual resilience $(r=-0.25, \mathrm{p}<0.01)$, as anticipated. Importantly, additional positive associations exist between caregiver burden at the 30-day follow-up and parent perceived stress, parent perceived child stress, COVID -specific family stressors, and depression symptomology ( $r$ s ranging from 0.35 to 0.64 , all $\mathrm{p}<0.01)$. Bivariate associations indicate child-parent relationship conflict at the 30-day follow-up was significantly negatively associated with baseline individual resilience and child-parent relationship closeness $(r s=-0.29$ and -0.38 , all $\mathrm{p}<0.01$ ). Additional positive associations exist between child-parent relationship conflict at the 30-day follow-up and parent perceived stress, parent perceived child stress, COVID-specific family stressors, and depression symptomology ( $r$ s ranging from 0.37 to 0.70 , all $\mathrm{p}<0.01$ ). Bivariate associations indicate child-parent relationship closeness at the 30-day follow-up was significantly positively associated with baseline individual resilience $(r=0.55, \mathrm{p}<0.01)$. Additional negative associations
Table 2 Bivariate correlations among variables of interest for the overall sample $(n=271)$

\begin{tabular}{lllllllll}
\hline & 1 & 2 & 3 & 4 & 5 & 6 & 7 & 7 \\
\hline Caregiver burden (T2) & - & & & & & & \\
Child parent relationship conflict (T2) & $.59^{* *}$ & - & - & & & & \\
Child parent relationship closeness (T2) & $-.28^{* *}$ & $-.38^{* *}$ & & & & & \\
Adult resilience (T1) & $-.25^{* *}$ & $-.29^{* *}$ & $.55^{* *}$ & - & & & \\
Parent perceived stress (T1) & $.47^{* *}$ & $.51^{* *}$ & $-.25^{* *}$ & $-.49^{* *}-$ & & \\
Perceived child stress (T1) & $.50^{* *}$ & $.70^{* *}$ & $-.58^{* *}$ & $-.41^{* *}$ & $.62^{* *}$ & - & \\
COVID-specific family stressors (T1) & $.35^{* *}$ & $.37^{* *}$ & -.08 & -.08 & $.40^{* *}$ & $.36^{* *}-$ & \\
Depression (T1) & $.64^{* *}$ & $.70^{* *}$ & $-.36^{* *}$ & $-.37^{* *}$ & $.73^{* *}$ & $.72^{* *}$ & $.47^{* *}-$ \\
\hline
\end{tabular}

${ }^{*} \mathrm{p}<.05, * * \mathrm{p}<.01$ 
exist between child-parent relationship closeness at the 30-day follow-up and parent perceived stress, parent perceived child stress, and depression symptomology ( $r \mathrm{~s}$ ranging from -0.25 to -0.58 , all $\mathrm{p}<0.01$ ). Given potential gender differences noted in the resilience literature, bivariate associations for resilience were run separately for male and female caregivers: male caregiver resilience was significantly positively associated with child parent relationship closeness $(r=0.42, p<0.01)$, as well as significantly negatively associated with caregiver burden, child parent relationship conflict, parent perceived stress, parent perceived child stress, COVID-specific family stressors, and depression symptomology ( $r$ s ranging from -0.42 to -0.70 , all $\mathrm{p}<0.01)$. Female caregiver resilience was significantly positively associated with child parent relationship closeness $(r=0.43, p<0.01)$, as well as significantly negatively associated with caregiver burden, child parent relationship conflict, parent perceived stress, parent perceived child stress, and depression symptomology ( $r$ s ranging from -0.24 to -0.54 , all $\mathrm{p}<0.01)$.

Independent samples t-tests were then conducted to assess for group differences based on parent gender, financial resources, and single-parent status. Several statistically significant group differences are evidence (see Table 3): male caregivers reported significantly higher rates at baseline of depression symptomology $(\mathrm{t}=2.53, \mathrm{p}<0.01)$, parent perceived child stress $(t=3.74, p<0.01)$, and significantly higher rates at the 30-day follow-up of child-parent relationship conflict $(\mathrm{t}=3.12, \mathrm{p}<0.01)$ and closeness $(\mathrm{t}=-4.59$, $\mathrm{p}<0.01$ ), whereas female caregivers reported significantly higher rates of individual resilience $(t=-1.97, p<0.05)$. Caregivers whose financial needs were not met reported significantly higher rates at baseline of depression symptomology $(\mathrm{t}=2.79, \mathrm{p}<0.01)$, parent stress $(\mathrm{t}=4.51, \mathrm{p}<0.01)$, and parent perceived child stress $(t=2.33, p<0.05)$, and significantly higher rates at the 30-day follow-up of child-parent relationship conflict $(\mathrm{t}=2.12, \mathrm{p}<0.05)$, whereas parents whose financial needs were met reported significantly higher rates at baseline of individual resilience $(\mathrm{t}=-3.76, \mathrm{p}<0.01)$. Partnered parents reported significantly higher rates at baseline of depression symptomology $(t=3.33, p<0.01)$, parent perceived child stress $(t=3.02, p<0.01)$, and significantly higher rates of child-parent relationship conflict $(t=2.26$, $\mathrm{p}<0.01)$ at the 30-day follow-up. Next, bivariate associations assessed differences on key variables by parent age: baseline reports of parent stress, parent perceived child stress, depression symptomology and COVID-specific family stressors were significantly negatively associated with parent age ( $r$ s ranging from -0.34 to -0.13 , all $\mathrm{p}<0.05$ ), and at the 30-day follow-up caregiver burden and child-parent relationship conflict was significantly negatively associated with parent age $(r s=-0.29$ and $-0.34, \mathrm{p}<0.01)$. Lastly, oneway ANOVA assessed differences on key variables by child focal age categories: parents of children ages 12 to 18 years

Table 3 Means, standard deviations, and significant group differences $(\mathrm{n}=271)$

\begin{tabular}{|c|c|c|}
\hline Variable & $\mathrm{M}(\mathrm{SD})$ & Significant group differences (effect size) \\
\hline Caregiver burden (T2) & $12.69(9.25)$ & Negatively associated with parent age $(r=-0.29)$ \\
\hline Child parent relationship conflict (T2) & $19.62(9.14)$ & $\begin{array}{l}\text { Reported more by men than women }(\mathrm{d}=0.38) \text {; } \\
\text { Reported more by caregivers whose financial needs were not met }(\mathrm{d}=0.26) \text {; } \\
\text { Reported more by partnered caregivers }(\mathrm{d}=0.33) \text {; } \\
\text { Negatively associated with parent age }(\mathrm{r}=-0.34)\end{array}$ \\
\hline Child parent relationship closeness (T2) & $30.23(4.71)$ & Reported more by women than men $(d=0.67)$ \\
\hline Adult resilience $(\mathrm{T} 1)$ & $68.62(11.63)$ & $\begin{array}{l}\text { Reported more by women than men }(d=0.24) \text {; } \\
\text { Reported more by caregivers whose financial needs were met }(d=0.46) \text {; }\end{array}$ \\
\hline Parent perceived stress (T1) & $16.12(7.58)$ & $\begin{array}{l}\text { Reported more by caregivers whose financial needs are not met }(\mathrm{d}=0.55) \text {; } \\
\text { Negatively associated with parent age }(\mathrm{r}=-0.22)\end{array}$ \\
\hline Perceived child stress (T1) & $23.96(7.11)$ & $\begin{array}{l}\text { Reported more by men than women }(\mathrm{d}=0.46) \text {; } \\
\text { Reported more by caregivers whose financial needs are not met }(\mathrm{d}=0.29) \text {; } \\
\text { Reported more by partnered caregivers }(\mathrm{d}=0.46) \text {; } \\
\text { Reported less for parents of } 12-18 \text { year-olds }(\mathrm{F}=5.26) \\
\text { Negatively associated with parent age }(\mathrm{r}=-0.34)\end{array}$ \\
\hline COVID-specific family stressors (T1) & $10.85(10.03)$ & Negatively associated with parent age $(r=-0.13)$ \\
\hline Depression (T1) & $19.49(17.95)$ & $\begin{array}{l}\text { Reported more by men than women }(\mathrm{d}=0.31) \text {; } \\
\text { Reported more by caregivers whose financial needs are not met }(\mathrm{d}=0.34) \text {; } \\
\text { Reported more by partnered caregivers }(\mathrm{d}=0.46) \text {; } \\
\text { Negatively associated with parent age }(\mathrm{r}=-0.34)\end{array}$ \\
\hline
\end{tabular}

Two-tailed independent samples T-tests were used for categorical group comparisons; One-way ANOVA was used for group categorizations with three or more groups (focal child age: birth to 5 years old, 6 to 11 years old, and 12 to 18 years old); bivariate correlations were used for continuous predictors (age). Standardized effect sizes for group difference tests rely on Cohen's d $(0.20=$ small, $0.50=$ medium, $0.80=$ large $)$; Pearson's $\mathrm{r}$ is used for all bivariate correlations $(.1=$ small, $.3=$ medium, $.5=$ large $)$ 
old perceived less child stress $(F=5.26, p<0.01)$ than parents of younger children (both those with 0 to 5 year-olds and 6 to 11 year-olds).

\section{Path Analysis}

The results of a multi-group path analysis organized by parent gender is presented (see Figs. 1 and 2, for female and male caregivers, respectively). The multi-group path model had good fit, $X^{2}(18)=70.10, p<0.01, C F I=.94$, RMSEA $=0.10$. Taken together, there is evidence of appropriate goodness of fit of the model to the data. The model Chi Square $p$ value was well less than 0.05; and the CFI had a value greater than 0.90 . The RMSEA is a bit high, but this may be an artifact of the complexity of the model. Some [79] report that the index can be positively biased toward higher values and that the bias is associated with both sample size and degrees of freedom. Given that there are relatively few degrees of freedom in the current multigroup model, the RMSEA might be expected to be positively biased in this instance.

The multi-group model indicated no significant association between the control (focal child age) or parent-child closeness and any endogenous variables (parent stress, parent perceived child stress, COVID-related family experiences and stressors, depression symptomology, child parent relationship conflict and caregiver burden), thus they were removed from the model.

\section{Individual Resilience}

As hypothesized, caregiver individual resilience was negatively associated with parent stress $(\beta=-0.44$ and -0.55$)$, and parent perceived child stress $(\beta=-0.46$ and -0.34$)$, for female and male caregivers, respectively. Parents who reported higher rates of individual resilience reported lower rates of parent stress and parent perceived child stress. Our findings further indicate female caregivers reported higher rates of parent stress overall than male caregivers, whereas male caregivers reported higher rates of parent perceived child stress overall than female caregivers. However, individual resilience was not significantly directly associated with COVID-specific family stressors $(\beta=-0.03$ and $-0.18, p=n s)$ or depression symptomology $(\beta=-0.04$ and $0.12, p=n s)$, nor with caregiver burden $(\beta=0.01$ and $-0.04, p=n s)$ or childparent relationship conflict $(\beta=-0.13$ and $0.02, p=n s)$ at the 30-day follow-up for either group of caregivers (females and males, respectively).

\section{Parent Stress}

As hypothesized, parent stress was significantly associated with depression symptomology $(\beta=0.52$ and 0.48$)$, for female and male caregivers, respectively. Parents who

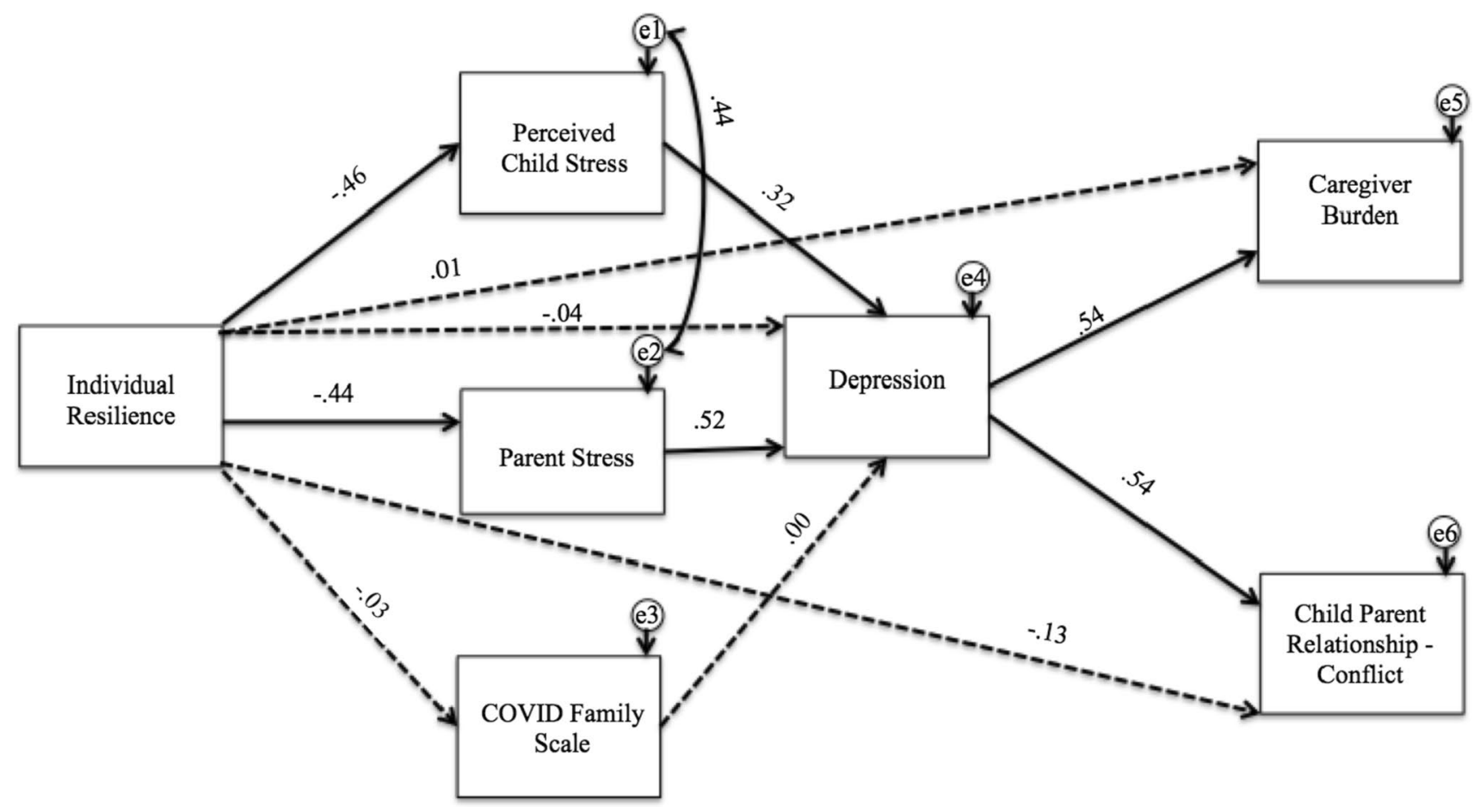

Fig. 1 Model of female caregivers' individual resilience with standardized path estimates $(n=124)$ 


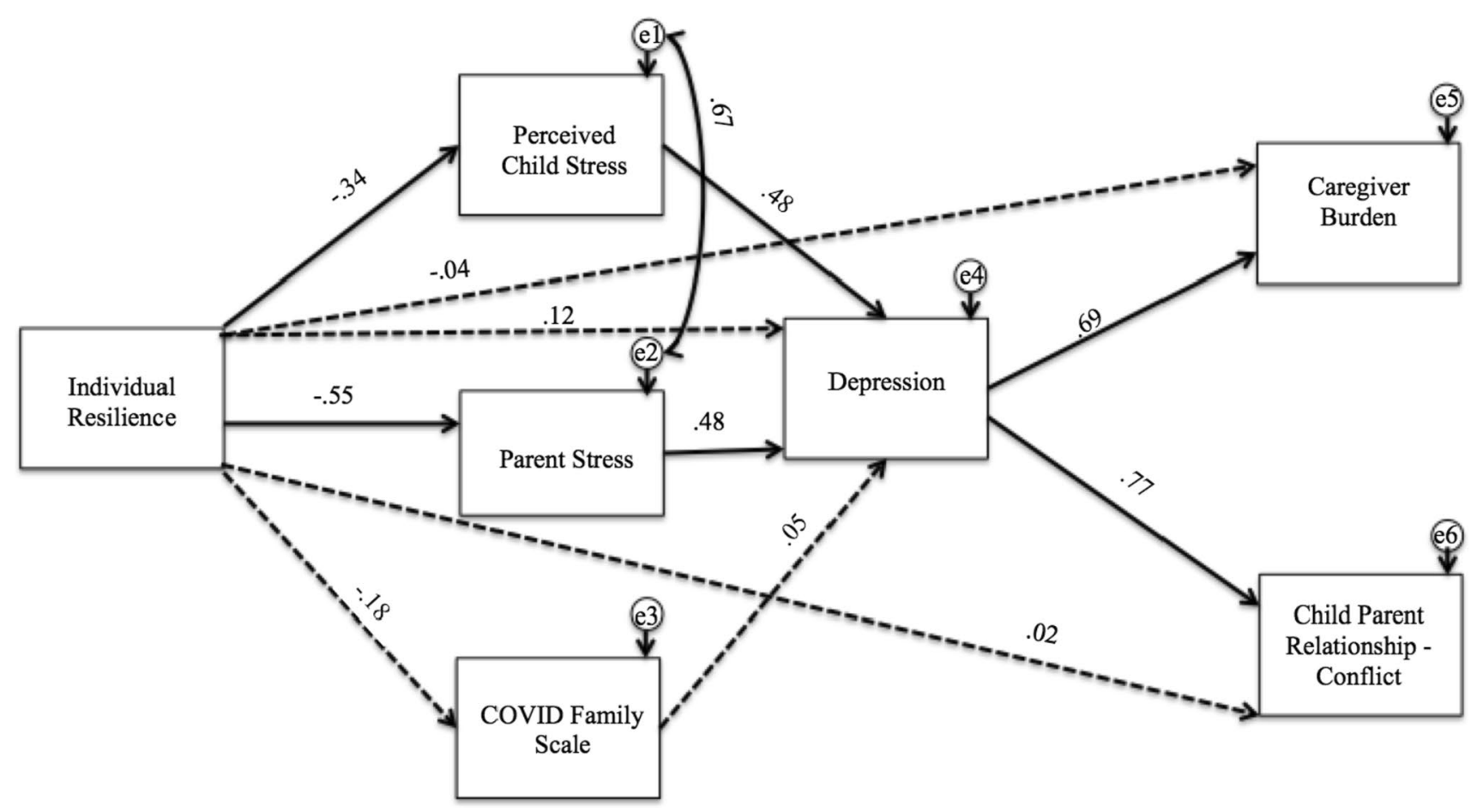

Fig. 2 Model of male caregivers' individual resilience with standardized path estimates $(n=147)$

reported higher rates of stress additionally reported higher rates of depression symptomology, with female caregivers reporting higher rates than their male counterparts.

\section{Parent Perceived Child Stress}

As hypothesized, parent reports of increased perceived child stress were significantly associated with depression symptomology $(\beta=0.32$ and 0.48$)$, for female and male caregivers, respectively. Parents who reported higher rates of stress additionally reported higher rates of depression symptomology, with male caregivers reporting higher rates than female caregivers.

\section{COVID-Specific Family Stressors}

Contrary to our hypothesis, COVID related family experiences and stressors did not significantly predict depression symptomology ( $\beta=0.00$ and $0.05, p=n s)$ for either group of caregivers.

\section{Depression}

As hypothesized, depression symptomology was significantly positively associated with caregiver burden $(\beta=0.54$ and $0.69, \mathrm{p}<0.01)$ and child-parent relationship conflict $(\beta=0.54$ and $0.77, p<0.01)$ at the 30 day follow-up for both groups of caregivers (females and males, respectively), suggesting caregivers with increased depression symptoms reported increased conflict in their parent-child relationship and caregiver burden, more so for male caregivers in our sample.

\section{Discussion}

Resilience at baseline was not directly associated with caregiver burden or child-parent relationship quality at the 30-day follow-up, but was indirectly linked to these outcomes through stress and depression pathways. Specifically, the models for both men and women indicate significant associations between baseline resilience and perceptions of both their own and their child's stress; these are both significantly linked to depression symptoms, which in turn is significantly associated with caregiver burden and conflict in the parent child relationship 30 days later. These findings are in line with the literature on stress appraisals and resilience [80-82] that notes that the resilience process can dampen the negative impact of perceived stress through impacting stress appraisals [83]: the more resilient the individual, the less severe the appraisal of the stressor both in terms of its potential disruption and the individual's ability to cope with the resulting stress. Participants reported on both their own and their perceptions of child stressors generally as well as self-reported stress appraisals specific to COVID-19 using a 2 week recall period at the end of April 2020, within only a 
few weeks following the first peak in 7-day average COVID cases in the US [35] and the shifts to daily activity enacted nationwide to stem the spread of the disease. Despite this stressful context, those participants with greater baseline resilience seem to have been buffered from experiencing both general and covid-specific stressors in heightened ways.

The final set of linkages between depression and both 30-day outcomes was stronger for men, suggesting that fathers' depression symptoms are producing stronger effects on their parenting experiences (caregiver burden and parent-child relationship quality) than effects reported by mothers. Fathers also reported higher rates of perceived child stress. This could result from shifts in fathers' roles since the onset of COVID-19, perhaps as a result of the record high national unemployment rates [36] that historically impact men moreso than women [84] in conjunction with shifts to remote work conditions. The current economic downturn has resulted in many parents spending more time at home attending to the needs of their children than prior to quarantine and work-from-home mandates [85]. Unemployment rates impacted women more than men during COVID-19 [86], and women reported worse mental health symptoms and caregiving burdens early in the pandemic compared to their male counterparts [27]. However, the impacts of parents working from home while co-located with young children needing care or older children learning from home were unprecedented. One possible explanation is that men found the novel competing demands of increased parenting support and balancing shifts in employment particularly taxing. An alternative explanation is that the long-standing history of disproportionate childcare burdens and employment inequities women face in the US [86, 87] has led women to build a greater set of protective factors and coping mechanisms for these strains.

For both mothers and fathers, results indicate a lack of significant direct pathways from resilience to caregiver burden or child-parent relationship quality; however, the linkage between resilience and parent stress and parent perceived child stress initiates a sequence of significant linkages to parent depression, and then to caregiver burden and parent-child relationship quality. Both mothers' and fathers' stress experiences were similar predictors of their reported rates of depression; similarly, for both mothers and fathers, resilience was a negative predictor of both personal and perceived child stress. In sum, stronger parent resilience was linked to fewer depression symptoms and less stress, contributing to lower conflict in the parent-child relationship and reduced caregiver burden, emphasizing previously reported impacts of resilience at the individual and family levels [38].

Notable significant group differences emerged for parents based on reports of having financial resources adequate to meet needs. Parents who reported not having the necessary financial resources to support their families also reported more parent-child conflict, as well as higher rates of parent stress and depression. As expected, reports of financial needs being met were associated with higher rates of parent resilience. The results of the present study, therefore, echo previous findings that financial security has an important influence on resilience $[10,88]$.

Significant group differences in mean scores were also evident based on partner/relationship status. Contrary to expectations, partnered parents reported increased rates of perceived child stress and depression. While additional data over a longer period of time, from a sample with a greater proportion of single parents is necessary to inform any causal inferences, it is possible that partnered caregivers who have been quarantined with their families have become more sensitive to the experiences of their partners and children, amplifying stress and depression rates as well as perceived child stress. Evidence from a meta-analysis of public health disasters necessitating quarantine [5] indicates that quarantine, particularly long quarantine, negatively impacts mental health. Given evidence from previous studies establishing significant links between parents' stress and depression, perceived child stress, and parent-child relationship quality, our results suggest spillover effects that occur when one family member's behavior or affect influences other members of the family system [51]. In this instance, we see evidence of a spillover effect wherein negative affect can transfer within a family system (i.e., negative affect in the parent is linked to perceived negative affect in the child). It is interesting to note the lack of a corresponding compensatory effect, as hypothesized, such that one might see with models that report significant paths to parent-child closeness [34]. In future studies, the addition of data on partners' mental health and relationship quality as reported by both co-parents would add valuable information on possible additional spill-over effects. For example, dyadic data would allow for the examination of crossover effects, where rather than a transfer of same-valanced affect from parent to child subsystems, crossover would indicate the transfer of affect or behavior between people (e.g., when the co-parenting stress experienced by one caregiver crosses over to impact the other partner's relationship with their child [51]). Interestingly, COVID-19 specific stressors did not have a significant effect on mental health outcomes within this sample. It is possible that parents' depression was more powerfully associated with general stress rates and perceived child stress (as reported on both forms of the Perceived Stress Scale) than with COVID-19 specific stressors because of the pervasiveness of the global pandemic. Individuals may see COVID-19-related stressors as universal, or transient, and related to this pandemic. This sense that COVID-19-related family stress is normative may lead individuals to place less emphasis or derive less meaning from on those stressors. 
Future researchers ought to examine links between COVID19-related stressors and ongoing reports of stress, or other indications of stress saturation.

Several limitations should be noted. While our sample was reasonably balanced by roughly equal numbers of men and women who reported a relatively normative income distribution, MTurk as an online platform still attracts users with higher education levels and access to Internet that may not be broadly generalizable. Furthermore, given evidence that individual characteristics like ethnic and racial identity impact resilience [10], efforts to recruit a more diverse sample are warranted. Additionally, including measures of parenting behavior during COVID-19 would enable further examination of families' socialization of coping strategies. Some [89] have found that parents report talking with their children about COVID-19 related viral transmission mitigation strategies, like hand washing, and social distancing, and are sensitive to their children's coping behaviors, such as playing video games, or modifying socialization patterns. Lastly, the inclusion of family functioning measures reported by additional family members-such as co-parents or child-reports-would increase understanding of family experiences across family subsystems.

Overall, it is evident that fathers, specifically, are struggling with a functional impairment of their parent-child relationships associated with their depression symptoms, as seen through the significant associations with caregiver burden and parent-child conflict. Given the needed adaptation in response to surging infection rates seen across geographic region [90], parents clearly need more resources and supports to manage personal and family mental health and well-being. Certainly, events that cause tumultuous shifts in routine and create unpredictability in a sense of security call for an increase in family assistance than spans both financial and socioemotional supports. Such is the case for the range of stressors associated with COVID-19 as families need continued support navigating drastic societal shifts with particular demands for mental health, financial, and child-care supports [85]. Each of these needs carries specific policy and practice implications - for example, adapting social welfare and mental health service provision to telehealth modalities, expansion of unemployment and pay check protection benefits, provision of safe and affordable childcare, and access to communication technologies to enable remote or virtual healthcare access, employment, and education opportunities for all families.

\section{Summary}

Parents and other caregivers report marked impacts on mental health as a result of the COVID-19 pandemic. Longitudinal associations from this national sample during the early months of the American pandemic indicate different patterns of resilience, stress, and parenting outcomes by parent gender. The extant literature indicates these effects may be result of on-going uncertainty concerning the quarantine and social distance guidelines in place to slow the spread of the virus, but which hamper access to psychosocial and pragmatic supports. Additionally, caregiver strains in particular may be the product of still-evolving work and family routines with steep implications for how families provide and care for the children in their homes. Multigroup path model results depict a series of linkages over a short, 30-day period that begin with connections between resilience and parent stress or parent perceived child stress, followed by linkages to parent depression and then caregiver burden and parent-child relationship quality. This final set of relationships between depression and both parenting outcomes were significantly stronger for men, who also reported higher rates of perceived child stress. These patterns indicate that fathers' depression symptoms and associated spill-over to perceived child stress is producing stronger effects on their parenting experiences than effects reported by mothers, leading to higher reports of conflict and caregiver burden.

\section{References}

1. Baral IA, Bhagawati KC (2019) Post Traumatic Stress Disorder and coping strategies among adult survivors of earthquake, Nepal. BMC Psychiatry 19(1):118

2. North CS (2016) Disaster mental health epidemiology: methodological review and interpretation of research findings. Psychiatry 79:130-146. https://doi.org/10.1080/00332747.2016.1155926

3. Layne SP, Hyman JM, Morens DM, Taubenberger JK (2020) New coronavirus outbreak: framing questions for pandemic prevention. Sci Transl Med 12(534):eabb1469. https://doi.org/10.1126/scitr anslmed.abb1469

4. Galea S, Coffey SF (2018) Social support moderates effects of natural disaster exposure on depression and posttraumatic stress disorder symptoms: effects for displaced and nondisplaced residents. J Trauma Stress 31:223-233

5. Brooks SK, Webster RK, Smith LE, Woodland L, Wessely S, Greenberg N, Rubin GJ (2020) The psychological impact of quarantine and how to reduce it: rapid review of the evidence. Lancet 395(10227):912-920. https://doi.org/10.1016/S0140-6736(20) 30460-8

6. Cluver L, Lachman JM, Sherr L et al (2020) Parenting in a time of COVID-19. Lancet 395:e64

7. Horesh D, Brown AD (2020) Traumatic stress in the age of COVID-19: a call to close critical gaps and adapt to new realities. Psychol Trauma Theory Res Pract Policy 12:331-335. https://doi. org/10.1037/tra0000592

8. Windle G (2011) What is resilience? A review and concept analysis. Rev Clin Gerontol 21:152-169

9. Bonanno GA (2004) Loss, trauma, and human resilience: have we underestimated the human capacity to thrive after extremely aversive events? Am Psychol 59(1):20-28. https://doi.org/10.1037/ 0003-066X.59.1.20

10. Norris FH, Friedman MJ, Watson PJ, Byrne CM, Diaz E, Kaniasty K (2002) 60,000 disaster victims speak: Part I. An 
empirical review of the empirical literature, 1981-2001. Psychiatry 65(3):207-239. https://doi.org/10.1521/psyc.65.3.207.20173

11. Bonanno GA, Galea S, Bucciarelli A, Vlahov D (2007) What predicts psychological resilience after disaster? The role of demographics, resources, and life stress. J Consult Clin Psychol 75(5):671

12. Bundick MJ, Yeager DS, King PE, Damon W (2010) Thriving across the life span. In: Overton WF, Lerner RM (eds) The handbook of life-span development, vol 1. Cognition, biology, and methods. John Wiley \& Sons, Somerset, NJ, pp 882-923

13. Masten AS (2007) Resilience in developing systems: progress and promise as the fourth wave rises. Dev Psychopathol 19:921. https://doi.org/10.1017/S0954579407000442

14. Gartland D, Bond L, Olsson CA et al (2011) Development of a multi-dimensional measure of resilience in adolescents: the Adolescent Resilience Questionnaire. BMC Med Res Methodol 11:134. https://doi.org/10.1186/1471-2288-11-134

15. Liebenberg L, Ungar M (Eds) (2009) Researching Resilience. University of Toronto Press. https://doi.org/10.3138/9781442697669

16. Masten AS (2001) Ordinary magic: resilience processes in development. Am Psychol 56(3):227-238

17. Bolt MA, Helming LM, Tintle NL (2018) The associations between self-reported exposure to the Chernobyl nuclear disaster zone and mental health disorders in Ukraine. Front Psych. https:// doi.org/10.3389/fpsyt.2018.00032

18. Fussell E, Lowe SR (2014) The impact of housing displacement on the mental health of low-income parents after hurricane Katrina. Soc Sci Med 113:137-144

19. Labarda CE, Jopson QDQ, Hui VK, Chan CS (2020) Long-term displacement associated with health and stress among survivors of typhoon Haiyan. Psychol Trauma. https://doi.org/10.1037/tra00 00573

20. Seto M, Nemoto H, Kobayashi N, Kikuchi S, Honda N, Kim Y, Kelman I, Tomita H (2019) Post-disaster mental health and psychosocial support in the areas affected by the great east japan earthquake: a qualitative study. BMC Psychiatry 19(1):1-3

21. Sprague CM, Kia-Keating M, Felix E, Afifi T, Reyes G, Afifi W (2015) Youth psychosocial adjustment following wildfire: the role of family resilience, emotional support, and concrete support. Child Youth Care Forum 44(3):433-450

22. Hawryluck L, Gold WL, Robimson S, Pogorski S, Galea S, Styra R (2004) SARS control and psychological effects of quarantine, Toronto, Canada. Emerg Infect Dis 10:1206-1212

23. Carleton NK (2016) Into the unknown: a review and synthesis of contemporary models involving uncertainty. J Anxiety Disord 39:30-43

24. Sweeny K (2018) On the experience of awaiting uncertain news. Curr Dir Psychol Sci 27:281-285. https://doi.org/10.1177/09637 21417754197

25. Ellis DM, Hudson JL (2010) The metacognitive model of generalized anxiety disorder in children and adolescents. Clin Child Fam Psychol Rev 13:151-163

26. Russell BS, Hutchison M, Tambling R, Tomkunas AJ, Horton AL (2020) Initial challenges of caregiving during COVID-19: caregiver burden, mental health, and the parent-child relationship. Child Psychiatry Hum Dev 51(5):671-682. https://doi.org/ 10.1007/s10578-020-01037-x

27. Russell BS, Hutchison M, Park CL, Fendrich M. Finkelstein-Fox L (2021) Short-term impacts of COVID-19 on family caregivers: Emotion regulation, coping, and mental health. J Clin Psychol. https://doi.org/10.1002/jclp.23228

28. Kerns CE, Elkins RM, Carpenter AL, Chou T, Green J, Comer J (2014) Caregiver distress, shared traumatic exposure, and child adjustment among area youth following the 2013 Boston marathon bombing. J Affect Disord 167:50-55
29. Maeda M, Oe M (2017) Mental health consequences and social issues after the Fukushima disaster. Asia Pac J Public Health 29:36S-46S

30. Kliewer W, Fearnow MD, Miller PA (1996) Coping socialization in middle childhood: tests of maternal and paternal influences. Child Dev 67(5):2339-2357

31. Shipman KL, Schneider R, Fitzgerald MM, Sims C, Swisher L, Edwards A (2007) Maternal emotion socialization in maltreating and non-maltreating families: implications for children's emotion regulation. Soc Dev 16:268-285

32. Juth V, Silver RC, Seyle DC, Widyatmoko CS, Tan ET (2015) Post-disaster mental health among parent-child dyads after a major earthquake in Indonesia. J Abnorm Child Psychol 43:1309_ 1318. https://doi.org/10.1007/s10802-015-0009-8

33. Kılıç C, Kılıç EZ, Aydın İO (2011) Effect of relocation and parental psychopathology on earthquake survivor-children's mental health. J Nervous Mental Dis 199:335-341. https://doi.org/10. 1097/NMD.0b013e3182174ffa

34. Masten AS, Narayan AJ (2012) Child development in the context of disaster, war, and terrorism: pathways of risk and resilience. Annu Rev Psychol 63:227-257

35. Dong E, Du H, Garnder L (2020) An interactive web-based dashboard to track COVID-19 in real time. Lancet 20:533-534. https:// doi.org/10.1016/S1473-3099(20)30147-X

36. United States Bureau of Labor Statistics (2020a). Unemployment rate. https://data.bls.gov/timeseries/LNS14000000

37. Deater-Deckard K (2008) Parenting stress. Yale University Press, New Haven

38. Masten AS (2018) Resilience theory and research on children and families: past, present, and promise. J Fam Theory Rev 10(1):12-31

39. White JM, Klein DM (2002) Family theories. Sage, Thousand Oaks

40. Granek L, Rosenberg-Yunger ZR, Dix D, Klaassen RJ, Sung L, Cairney J, Klassen AF (2012) Caregiving, single parents and cumulative stresses when caring for a child with cancer. Child Care Health Dev 40:184-194. https://doi.org/10.1111/cch.12008

41. Martinez-Marcos M, De la Cuesta-Benjumea C (2015) Women's self-management of chronic illnesses in the context of caregiving: a grounded theory study. J Clin Nurs 24:1557-1566

42. Briggs-Gowan MJ, Carter AS, Schwab-Stone M (1996) Discrepancies among mother, child, and teacher reports: examining the contributions of maternal depression and anxiety. J Abnorm Child Psychol 24:749-765

43. Schechter DS, Willheim E, Hinojosa C, Scholfield-Kleinman K, Turner JB, McCaw J et al (2010) Subjective and objective measures of parent-child relationship dysfunction, child separation distress, and joint attention. Psychiatry 73(2):130-144

44. Taraban L, Shaw DS (2018) Parenting in context: revisiting Belsky's classic process of parenting model in early childhood. Dev Rev 48:55-81

45. Van Ee E, Kleber R, Mooren TTM (2012) War trauma lingers on: associations between maternal posttraumatic stress disorder, parent-child interaction, and child development. Infant Ment Health J 33(5):459-468. https://doi.org/10.1002/imhj.21324

46. Russell BS, Tambling R, Horton AL, Hutchison M, Tomkunas AJ (2021) Clinically significant depression among parents during the COVID-19 pandemic: examining the protective role of family relationships. J Couple Family Psychol. https://doi.org/10.1037/ cfp0000175

47. Cobham VE, McDermott B, Haslam D, Sanders MR (2016) The role of parents, parenting and the family environment in children's post-disaster mental health. Curr Psychiatry Rep 18(6):53. https:// doi.org/10.1007/s11920-016-0691-4 
48. Lambert JE, Holzer J, Hasbun A (2014) Association between parents' PTSD severity and children's psychological distress: a meta-analysis. J Trauma Stress 27:1-9

49. Darling N, Steinberg L (1993) Parenting style as context: an integrative model. Psychol Bull 113:487-496

50. Labella MH, Narayan AJ, Masten AS (2016) Emotional climate in families experiencing homelessness: associations with child affect and socioemotional adjustment in school. Soc Dev 25:304-321

51. Nelson JA, O'Brien M, Blankson AN, Calkins SD, Keane SP (2009) Family stress and parental responses to children's negative emotions: Tests of the spillover, crossover, and compensatory hypotheses. J Fam Psychol 23:671-679

52. Bartneck C, Deunset A, Moltchanova E, Zawieska K (2015) Comparing the similarity of responses received from studies in Amazon's Mechanical Turk to studies conducted online and with direct recruitment. PLoS ONE 10:1-23

53. Sheehan KB, Pittman M (2016) Amazon's mechanical Turk for academics: the HIT handbook for social science research. Melvin \& Leigh, Irvine

54. Engle K, Talbot M, Samuelson KW (2019) Is Amazon's Mechanical Turk (MTurk) a comparable recruitment source for trauma studies? Psychol Trauma Theory Res Pract Policy 12:381-388. https://doi.org/10.1037/tra0000502

55. McCredie MN, Morey LC (2019) Who are the Turkers? A characterization of MTurk workers using the personality assessment inventory. Assessment 26(5):759-766

56. Ophir Y, Sisso I, Asterhan CS, Tikochinski R, Reichart R (2020) The Turker blues: Hidden factors behind increased depression rates among Amazon's Mechanical Turkers. Clinical Psychological Science 8(1):65-83

57. Mortensen K, Hughes TL (2018) Comparing Amazon's Mechanical Turk platform to conventional data collection methods in the health and medical research literature. J Gen Intern Med 33:533-538

58. Chandler J, Shapiro D (2016) Conducting clinical research using crowdsourced convenience samples. Annu Rev Clin Psychol $12: 53-81$

59. Liebenberg L, Moore J (2018) A social ecological measure of resilience for adults: the RRC-ARM. Soc Indic Res 136(1):1-19

60. Cohen S, Kamarck T, Mermelstein R (1983) A global measure of perceived stress. J Health Soc Behav 24:386-396

61. Beaumont JL, Havlik R, Cook KF, Hays RD, Wallner-Allen K, Korper SP et al (2013) Norming plans for the NIH toolbox. Neurology 80:S87-S92

62. Salsman JM, Butt ZA, Pilkonis PC, Cyranowski JJ, Zill NR, Hendrie HK et al (2013) Emotion assessment using the NIH Toolbox. Neurology 80:S76-S86

63. Park CL, Russell BS, Fendrich M, Finkelstein-Fox L, Hutchison M, Becker J (2020) Americans' Covid-19 stress, coping, and adherence to CDC guidelines. J Gen Intern Med. https://doi.org/ 10.1007/s11606-020-05898-9

64. Sytsma S, Kelley E, Wymer M (2001) Development and initial validation of the child routines inventory. J Psychopathol Behav Assess 23:241-251

65. Tambling RR, Russell BS, Park CL, Fendrich M, Hutchison M, Horton AL, Tomkunas AJ (2020) Measuring cumulative stressfulness: psychometric properties of the COVID-19 Stressors Scale. Health Educ Behav 48:20-28. https://doi.org/10.1177/10901 98120979912

66. Spitzer RL, Kroenke K, Williams JB, Löwe B (2006) A brief measure for assessing generalized anxiety disorder: the GAD-7. Arch Intern Med 166:1092-1097

67. Bech P, Rasmussen NA, Olsen LR, Noerholm V, Abildgaard W (2001) The sensitivity and specificity of the Major Depression Inventory, using the Present State Examination as the index of diagnostic validity. J Affect Disord 66:159-164
68. American Psychiatric Association (2013) Depression disorders. In: Diagnostic and statistical manual of mental disorders, 5th edn. American Psychiatric Association, Washington, DC

69. Olsson CA, Bond L, Burns JM, Vella-Brodrick DA, Sawyer SM (2003) Adolescent resilience: a concept analysis. J Adolesc 26(1):1-11. https://doi.org/10.1016/s0140-1971(02)00118-5

70. Graessel E, Berth H, Lichte T, Grau H (2014) Subjective caregiver burden: validity of the 10-item short version of the Burden Scale for Family Caregivers BSFC-s. BMC Geriatr 14:23. https://doi. org/10.1186/1471-2318-14-23

71. Driscoll K, Pianta RC (2011) Mothers' and fathers' perceptions of conflict and closeness in parent-child relationships during early childhood. J Early Child Infant Psychol 7:1-24

72. Arbuckle JL (2019) Amos (Version 26.0) [Computer Program]. IBM SPSS, Chicago

73. Endendijk J, Groeneveld M, Bakerman-Kranenburg M, Mesman J (2016) Gender-differentiated parenting revisited: meta-analysis reveals very few differences in parental control of boys and girls. PLoS ONE 11:e0159193. https://doi.org/10.1371/journal.pone. 0159193

74. Tamres LK, Janicki D, Helgeson VS (2002) Sex differences in coping behavior: a meta-analytic review and an examination of relative coping. Pers Soc Psychol Rev 6:2-30. https://doi.org/10. 1207/S15327957PSPR0601_1

75. Hayduk LA, Cummings GC, Boadu K, Pazderka-Robinson H, Boulianne S (2007) Testing! Testing! One, two, three: testing the theory in structural equation models! Personality Individ Differ 42(5):841-850

76. Barrett PT (2007) Structural equation modelling: adjudging model fit. Person Individ Differ 42(5):815-824. https://doi.org/10.1016/j. paid.2006.09.018

77. Bentler PM (1990) Comparative fit indexes in structural models. Psychol Bull 107:238-246. https://doi.org/10.1037/0033-2909. 107.2.23

78. Steiger JH (1990) Structural model evaluation and modification: an interval estimation approach. Multivar Behav Res 25:173-180

79. Hooper D, Coughlan J, Mullen M (2008) Structural equation modelling: guidelines for determining model fit. Electron J Bus Res Methods 6(1):53-60

80. Kalisch R, Baker DG, Basten U, Boks MP, Bonanno GA, Brummelman E et al (2017) The resilience framework as a strategy to combat stress-related disorders. Nat Hum Behav 1:784-790. https://doi.org/10.1038/s41562-017-0200-8

81. Lazarus RS, Folkman S (1987) Transactional theory and research on emotions and coping. Eur J Pers 1:141-169

82. Rutter M (2012) Resilience as a dynamic concept. Dev Psychopathol 24:335-344

83. Velichkovsky BB (2009) Primary and secondary appraisals in measuring resilience to stress. Psychol Russ 2:539-563

84. Sahin A, Song J, Hobjin B (2010) The unemployment gender gap during the 2007 recession. Curr Issues Econ Financ 16:1-7

85. Prime H, Wade M, Browne DT (2020) Risk and resilience in family well-being during the COVID-19 pandemic. Am Psychol 75:631-643

86. Bateman N, Ross M (2020) Brookings institute gender equality series: why has COVID-19 been especially harmful for working women. https://www.brookings.edu/essay/why-has-covid-19been-especially-harmful-for-working-women/

87. United States Bureau of Labor Statistics (2020b). American Time Use Survey. https://www.bls.gov/tus/tables/a6-1519.htm

88. Black K, Lobo M (2008) A conceptual review of family resilience factors. J Fam Nurs 14(1):33-55. https://doi.org/10.1177/10748 40707312237

89. Tambling R, Tomkunas AJ, Russell BS, Hutchison M, Horton AL (2020) Thematic analysis of parent-child conversations about 
COVID-19: "Playing it safe". J Child Fam Stud. https://doi.org/ 10.1007/s10826-020-01889-w

90. Johns Hopkins University Center for Systems Science and Engineering (2020) COVID-19 dashboard. https://www.arcgis.com/ apps/opsdashboard/index.html\#/bda7594740fd40299423467b4 $8 \mathrm{e} 9 \mathrm{ecf6}$
Publisher's Note Springer Nature remains neutral with regard to jurisdictional claims in published maps and institutional affiliations. 